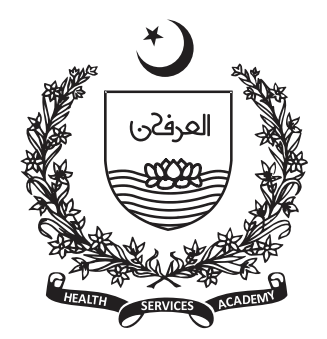

1PhD Student (Biological Sciences), Aga Khan University, Karachi, Pakistan

2Senior Instructor (Research), Department of Surgery, Aga Khan University, Karachi, Pakistan

${ }^{3}$ Assistant Professor (Research) and Head of Manuscript and Grants Division, Office of Academia and Research in Surgery (OARS)

Department of Surgery, Aga Khan University, Karachi, Pakistan

Corresponding Author: Saleema Mehboob Ali Email:

saleema.mehboob@scholar. aku.edu

\section{Pancreatic Cancers: A Review of Studies from Pakistan and Comparison with Global Trends}

\author{
Saleema Mehboob Ali1 ${ }^{1}$, Yumna Adnan², S.M. Adnan Ali ${ }^{3}$
}

\section{Abstract}

The objective of this review article is to discuss clinical data from studies on Pakistani pancreatic cancer population and its comparison with international data. This review article will allow researchers to identify gaps in the data and hence to design further research in order to address these gaps accordingly.

Search was carried out via Google Scholar, PubMed and PakMediNet search engines using keywords "Pancreatic cancers", "Pancreatic adenocarcinoma" and "Pakistan".

Pancreatic cancers are one of the most lethal cancers globally. Statistics show that $97.8 \%$ of cases of pancreatic cancers in Pakistan have led to mortality. For various cancers around the world, epidemiological data has been used for development of diagnostic tools and therapies. Despite the alarming situation of pancreatic cancers in Pakistan, only limited work has been done in this area. Majority of the studies are based exclusively on reporting of clinical data. Pakistan has not been able to even gather sufficient data to proceed research based on association of molecular aspects of tumor with clinical characteristics. On the contrary, researchers, scientists and clinicians from other parts of the world are already working on association studies, prediction of prognosis, treatment selection and improving the survival of their population.

It is an immense need of the situation that molecular based studies are carried out on Pakistani pancreatic cancer population so that diagnosis, prognosis, treatment and survival of these patients can be made better.

Keywords: Pancreatic cancers, clinical features, diagnosis, survival, treatment

\section{Introduction}

$\mathrm{P}$ ancreatic cancer is among the top 10 most fatal cancers worldwide. In comparison to 2012, there is more than two fold rise in the incidence of pancreatic cancers in 2020 in Pakistan with a $97.4 \%$ mortality rate. According to Globocan report, it has been predicted that by 2040, incidence of pancreatic cancers in Pakistan will become approximately doubled with high numbers of mortality $(1,2)$.

Anatomically, pancreatic cancers can be divided into two broad categories; exocrine and endocrine tumors. According to reports, $93 \%$ of all pancreatic tumors worldwide are of exocrine origin, majority (9 out of 10) of which are adenocarcinoma. Pancreatic adenocarcinoma is the malignancy that arises from the epithelial linings of the pancreatic duct. Other exocrine tumors include acinar cell carcinoma, intraductal papillary mucinous neoplasm and mucinous cystic neoplasm (often leads to invasive adenocarcinoma). A minority of pancreatic cancers are of endocrine origin. Endocrine tumors are also generally termed as Pancreatic Neuroendocrine Tumors (PNETs). PNETs include gastrinoma, glucagonoma, insulinoma, somatostatinoma, VIPoma and Non-functional islet 
cell tumor (3). Approximately $10 \%$ of all pancreatic cancers are found to be hereditary (4).

Globally, analysis of epidemiological and clinical data from various cancers have been used for elucidating association with molecular aspects of the disease and development of diagnostic tests, prognosis prediction tools and targeted therapies. From Pakistan, only 23 studies have been published on pancreatic cancers in the last 14 years. Most of these studies are hospital based and focused on the reporting of clinical data (mainly tumor size, grade and overall survival) and effectiveness of surgical methods in pancreatic cancers. Among these, there are only three studies which included molecular based aspects. One study reports expression of selected cell surface markers detected through immunohistochemistry (IHC) performed on pancreatic tumor tissues. (5). Moreover, other two investigations are based on bioinformatics and analysis of serum Carcinoembryonic Antigen (CEA) for pancreatic cancer diagnosis respectively (6). Results from all three studies remained inconclusive due to smaller sample size (22 for IHC while only one for bioinformatics and CEA analysis respectively). No other study with aim to analyze molecular aspects of pancreatic cancers have been published from Pakistan. Figure 1 shows pancreatic cancer based research publications from different parts of Pakistan. Six review articles have also been published that include the role of mi-RNA as biomarkers, role of diabetes and diagnosis in pancreatic cancers (7-11).

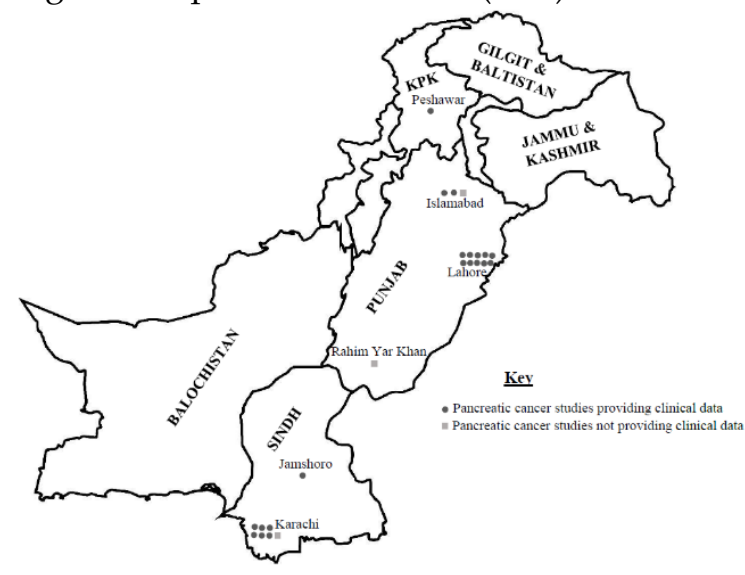

Figure 1: Pancreatic cancer-based research publications from Pakistan

\section{Methodology}

Search was carried out via Google Scholar, PubMed and PakMediNet search engines using keywords
"Pancreatic cancers", "Pancreatic adenocarcinoma" and "Pakistan". All the original research articles based on Pakistani pancreatic cancer population were included for consideration. There were 23 such articles found at the time of review. The year range of the articles found as per the above mentioned inclusion criteria was from 2004 to 2018 . No article was found from the years 2019 and 2020.

\section{Results}

\section{Clinical Characteristics:}

\section{Risk Factors:}

Several epidemiological investigations have shown strong evidence that environmental risk factors play a substantial role in origination and progression of various cancers. For instance, smoking, red meat consumption and use of tobacco/alcohol pose significant attributable risk to lung, colorectal and esophageal cancers respectively. Exposure to ultraviolet radiations is associated with $90 \%$ risk of squamous skin cancers (12). According to cancer data published in the UK, 3 to 10 cancers in 100 cancer cases are inherited (13).

Comorbidities also play a significant contribution in cancer occurrence. Medicare beneficiaries' data from the United States shows that four in ten of all 65 years or older cancer patients have at least one recorded chronic condition. Among these, 15\% of these cancer patients have two or more recorded chronic health problems. According to clinical records' investigation, chronic hepatitis B and C are causes of $80 \%$ of all hepatocellular carcinomas. Diabetes has also been associated with an elevated risk of numerous cancers including liver, pancreatic, breast, endometrial and colorectal cancers. It has been experimentally demonstrated that certain biological pathways directly link diabetes with cancers $(12,14)$. It is evident from these reports that a variety of risk factors play a role in cancer pathology and hence should be studied in various populations.

Globally, risk factors associated with pancreatic cancers include diabetes type II, smoking, high Body Mass Index (BMI), alcohol and processed meat consumption. Diabetes type II patients have 1.8 fold increased risk of pancreatic cancers while individuals with at least two first degree relatives diagnosed with pancreatic cancers are at double risk. Literature shows that smokers have 2 times higher risk of developing pancreatic cancers than non-smokers. Patients with chronic pancreatitis were found to be at higher risk of 
pancreatic cancers as compared to the general population (4).

In data obtained from Pakistan, only one study that was published from Aga Khan University in 2004 reports the risk factors in pancreatic cancer patients. In this study, three risk factors were taken into consideration; diabetes mellitus, smoking and chronic pancreatitis. The said study had data for only 14 patients. Other pancreatic cancer related risk factors such as BMI, processed meat consumption, alcohol intake and family history were not included (15).

It is important to study cancer specific risk factors as tumorigenesis is a multi-step process and several elements like environment, genetic predisposition, lifestyle and co-morbidities play their role.

\section{Anatomic Location of Pancreatic Tumor:}

The anatomic location of pancreatic tumor has been suggestive of the prognosis and survival of the pancreatic cancer patients. The majority pancreatic tumors arise in the head of pancreas while only 20$25 \%$ occur in the body and tail combined (BT). Tumors located in the body and tail have been associated with poorer survival as compared to tumors in the head. One reason for this might be relatively late diagnosis of BT tumors. Patients with pancreatic head tumors often present jaundice while those with BT tumors have pain and weight loss complaints which are comparatively general and less critical symptoms (16). Body and tail tumors have been correlated with surgical unresectability, early invasion and poor differentiation (17).

According to the Surveillance, Epidemiology and End Results (SEER) report, the commonest site for pancreatic tumor diagnosis in the USA was head consisting of $72.36 \%$ pancreatic tumors. Tumors diagnosed in the body and tail of the pancreas consisted of $11.72 \%$ and $15.90 \%$ respectively (18).

The cumulative number of pancreatic cancer patients investigated in various Pakistani studies is 1330. Out of these, tumor site data is available for 909 patients $(68.35 \%)$. The most common site of tumor origin was the head of the pancreas (37.5\%). There were $78(8.6 \%)$ tumors that originated from the pancreatic body and/or tail $(5,19-26)$.

\section{Type of Pancreatic Cancer:}

According to international data, $93 \%$ of all pancreatic tumors are exocrine while a small percentage of cases are of endocrine origin (3). In addition to the tumor site, an important aspect of classification of tumors globally has been the molecular basis (27).

Among studies from Pakistan, out of 23, there were only 6 investigations which categorized the type of pancreatic tumor. The commonest type of tumor diagnosed was adenocarcinoma which consist of $70.80 \%$ of all tumors with known types. Other tumors were neuroendocrine, Gastrointestinal Stromal Tumors (GIST) or benign masses. $(23,24,26,28-30)$.

\section{Complains/ Complications on Presentation of Disease and Post-Operation:}

Complaints on presentation at hospital differ based on the origin of the disease. Investigations showed that patients with a tumor in the pancreatic body and/or tail mostly had abdominal pain and weight loss while those with tumors in the pancreatic head mostly presented jaundice. This may be because of the obstruction of bile duct which is present in close vicinity to the pancreatic head. Other accompanying symptoms in patients with pancreatic head tumor are pruritus, dark urine and pale stool (16).

Studies from different parts of the world have shown the analysis of a wide range of symptoms at diagnosis of pancreatic cancers in addition to the common symptoms i.e. jaundice, abdominal pain and weight loss. The range of other symptoms include swelling and cramp around the stomach, digestive disorders, back pain, fatigue, fever, itching, chills, nausea, insomnia, depression and poor appetite. Among these back pain and fatigue were found significantly associated with pancreatic cancers. A range of postoperative complications have also been studied (31). Major post-operative complications include pancreatic fistula, delayed gastric emptying and haemorrhage in the surrounding organs. These post-operative complications were correlated with reduced survival in patients with malignant disease. Studies have also focused on post-operative psychological changes in pancreatic cancer patients (32).

Among studies on Pakistani pancreatic cancer patients, complaints on disease presentation at hospital were reported by 8 researches while postoperation complications were investigated by only 5 studies. Combining both these provides data for 516 patients $(38.79 \%)$. The most common complaints on disease presentation found in these studies were abdominal pain, jaundice and weight loss while the commonest complication post-operation was pancreatic leakage $(15,21,23,26,28,29,33-35)$. 


\section{Diagnostic and Disease Progression Test History:} Pancreatic cancer is usually diagnosed in advanced stages when invasion and often metastasis has occurred. This is due to the complex location of the pancreas in the body, surrounded by various organs like stomach, liver and gallbladder. Other reasons for late diagnosis may be the appearance of general symptoms (like jaundice, abdominal pain and weight loss) and the absence of any sensitive diagnostic test for pancreatic cancers (3). Ultrasound and Computed Tomography (CT) scans are used for diagnostic purposes which create a high probability of false positive results due to the complex anatomical location of the pancreas. Researches have shown that Endoscopic Ultrasound (EUS) has a higher sensitivity than ordinary ultrasound and CT scans and can detect less than $2 \mathrm{~cm}$ pancreatic lesions (18). Currently in Pakistan, EUS is being used only at Shaukat Khanum Memorial Hospital and Research Center, Lahore, Sindh Institute of Urology and Transplantation, Karachi and Aga Khan University Hospital, Karachi. Global data shows that research based on noninvasive diagnostic tests with higher sensitivities are being carried out. Serum Cancer Antigen (CA-19) has been approved by FDA as a marker for pancreatic cancer management (36). There is no study from Pakistan that has reported the use of this marker.

Data from Pakistan shows six studies have recorded the diagnostic test performed on pancreatic cancer patients. Test history for $146(10.98 \%)$ patients was available. The commonest diagnostic technique used was Ultrasound (81 patients - 55.47\%). Among these, 30 patients underwent EUS. The second most common technique was CT scan (58 patients - 39.72\%) $(15,28$, 33-35, 37).

Only one study determined patients' disease progression through Eastern Cooperative Oncology Group (ECOG) performance status. Out of a total of 101 patients under investigation, status of $84.2 \%$ patients was from 0 to 2 (i.e. from fully active to being able to carry daily activities) while that of $15.8 \%$ patients were between 3 to 4 (i.e. from $50 \%$ confined to bed to fully disabled) on the ECOG standard scale (26).

\section{Tumor Grade:}

Grading of tumors is based on its cellular differentiation. Generally, it is divided in three categories i.e. well differentiated, moderately differentiated and poorly differentiated, depending on resemblance of tumor cells to normal cellular morphology in the specific organ (38). As the tumor progresses from well differentiated to poorly differentiated, the histology appears with greater number of darkly stained nuclei with lesser stroma in between. Cancer grade has been used as one of the important diagnostic and prognostic markers in several cancers including squamous cell carcinoma, cervical cancers and pancreatic cancers. Higher grade cancers have also been associated with poor survival of the patient (39).

Pooling data from SEER and other studies from the western world, moderately differentiated pancreatic cancer is diagnosed with the highest frequency $(83 \%)$ in the western part of the world $(18,40)$.

It has been generally observed that several studies from Pakistan have not included tumor grade among the reported clinical data. Among all 23 studies, only seven studies from Pakistan reported pancreatic tumor grades. $34.5 \%$ patients were found to be well differentiated, $53.2 \%$ moderately differentiated and $12.3 \%$ were poorly differentiated. One study also reported the relation of grade with survival of the patients $(55.5 \%$ patients with grade $2,40.7 \%$ with grade 1 and $3.8 \%$ with grade 3 were alive till last follow up - 2 years after date of disease diagnosis). $(19,21,22,24,28,29,37)$.

\section{Cancer Stage:}

Cancer stage is a depiction of spread of cancerous cells in a patient's body. Generally, it is divided into four stages depending on the extent of its spread: stage 1 being localized and stage 4 being metastasized (41). Advanced stage is associated with poor patient survival in many cancers including pancreatic cancers. Five year survival rate of pancreatic cancer patients with localized, regional and metastatic disease is $37 \%$, $12 \%$ and $3 \%$ respectively (3). 
SEER database and other studies representing European world show that $54.8 \%$ of all pancreatic cancer cases are diagnosed at stage 2 while $26.6 \%$ cases are diagnosed at stage 4 . Only $8.74 \%$ cases are diagnosed at stage $1(18,40)$.

In total six studies from Pakistan report pancreatic tumor stage. As per the data from these studies, the majority $(53 \%)$ were stage 3 and 4 tumors $(5,19,22,24$, $28,29)$.

\section{Invasion, Metastasis and Relapse:}

Late diagnosis and non-resectability are among the factors known for causing early invasion and metastasis of pancreatic cancers. Moreover, studies have shown that pancreatic tumors even metastasize after surgical removal. This is attributed to the aggressive nature of pancreatic tumors (42).

Following are the status of invasion, metastasis and relapse from the data obtained from Pakistani studies:

\section{Perineural Invasion:}

Only three studies report perineural invasion status in pancreatic cancers. Majority cases included in these studies were not detected with perineural invasion $(84.8 \%)(5,24,28)$.

\section{Lymph Node Invasion:}

Four investigations included lymph node invasion status in their study. $49.4 \%$ patients were found with positive lymph node invasion $(5,19,28,29)$.

\section{Vascular Invasion:}

Only two studies report vascular status. $45.6 \%$ pancreatic cancer patients had positive vascular invasion status. One study mentions the types of invaded vessels which were superior mesenteric artery, coeliac artery and common hepatic artery $(28$, $35)$.

\section{Metastasis and Relapse:}

Metastasis and relapse status in pancreatic cancer patients have been reported by four studies. The commonest site of metastasis is liver $(38.18 \%)$ while the second most common site is lungs (16.36\%). Duration between surgery and relapse is described by only one study. According to the follow-up data the mean duration between surgery and relapse was 12.33 months $(21,28,29,33)$.

\section{Survival:}

Survival of pancreatic cancer patients is affected by several factors. Among these, the most prominent is the late diagnosis of the disease. Due to delayed diagnosis, tumors are usually detected at an advanced stage which is associated with decreased survival of cancer patients.

From Pakistan, eight studies have reported survival of pancreatic cancer patients. These studies show that two year survival of pancreatic cancer patients is $72.5 \%$. Apart from these eight studies, another research has been published on the survival of pancreatic cancer patients treated with gemcitabine and folfirinox respectively. Results show that 1 year survival with folfirinox and gemcitabine treatment was $26 \%$ and $17 \%$ respectively $(5,15,19,22,24,28,33$, 43).

According to the published literature, the global 5year survival in pancreatic cancer is 6\% (ranging from 2 to $9 \%$ ). Late diagnosis has contributed immensely to a lower survival rate of these patients. Over the years, limited improvement in survival rate has been observed. According to the Northern Ireland Cancer Registry 5-year survival of these patients was 2.5\% and 5.2\% between 1993-1999 and 2005-2009 respectively (44).

Research and clinical trials are being conducted (mostly in western countries) in order to increase the survival of the patients but unfortunately the progress has been slow.

\section{Current Therapeutic Strategies:}

Among researches published from Pakistan, 12 studies report surgical details of pancreatic cancer patients. The commonest procedure was Whipple's resection which was performed in $79.5 \%$ of the patients $(5,15$, 19, 21, 24, 25, 28, 29, 33, 34, 37, 43). Chemotherapy and radiotherapy status of Pakistani pancreatic cancer population is one of the least known. Only four studies have included therapy status in their studies. According to these studies, $49.1 \%$ patients received chemotherapy, $4.09 \%$ patients received chemoradiotherapy while remaining received merely radiotherapy. According to these studies, chemotherapeutic agents used at Pakistani hospitals are gemcitabine, folfirinox and capecitabine which is conventional treatment for pancreatic cancers $(22,26$, 28 , 29). Currently, no pancreatic cancer drug clinical trial is being conducted in Pakistan. 
International literature show standard treatment includes surgical resection of the tumor followed by chemotherapy (Gemcitabine in combination with capecitabine, 5-fluorouracil or leucovorin). However, data demonstrates that due to late diagnosis, most pancreatic tumors fall in the category of borderline resectable or unresectable. Treatment given to these patients is chemotherapy or radio-chemotherapy (45). Globally, several clinical trials are being conducted in order to explore new therapeutic strategies that could play a role in better management and survival of pancreatic cancer patients. Programmed Death protein 1 and Programmed Death Ligand 1 (PD-1/PD-L1) are being used as an important marker for targeted drug development. On-going trials include the effect of durvalumab and nivolumab (targeted drugs for PD$1 /$ PD-L1) in pancreatic cancers. Both trials are currently in phase II (46). Other trials include the combined role of targeted drugs and chemotherapy in pancreatic cancers for example; bortezomib and carboplatin, lapatinib and capecitabine etc (46). Till date, erlotinib, pembrolizumab and olaparib are only Food and Drug Administration (FDA) approved targeted drugs to be used in pancreatic cancers with EGFR, PD-L1 over expression and defined $B R C A 1 / B R C A 2$ germline mutations respectively (47, $48)$.

\section{Discussion}

\section{Molecular Aspects of Pancreatic Cancers:}

Cancer is a complex and heterogeneous genetic disease. Investigations have demonstrated that cancers arising from the same anatomical site may also differ based on their genetic profiles. Molecular subtyping of cancers allow pooling of various cancers that harbor similar molecular characteristics and hence similar clinical features. Molecular aspects of various cancers are now being used as diagnostic and prognostic markers. Moreover, these are also being utilized for development of targeted therapies (49).

Researchers from all over the world especially developed regions have attempted to identify most prevalent genetic mutations in pancreatic cancers. Among these researches, the most widely detected mutations are in the KRAS gene which signals cells for proliferation and differentiation. Pancreatic Ductal Adenocarcinoma is known to be one of the most RAS addicted cancers as it possess nearly $100 \%$ RAS gene mutation frequency. Studies have correlated KRAS mutations with aggressive nature of pancreatic adenocarcinoma and shorter overall survival of these patients $(50,51)$. Other frequently detected mutated genes in pancreatic cancers include CDKN2A, SMAD4, BRAF, TP53, GNAS, RB1, RET, MPL, EGFR and NOTCH1. These mutations have also been significantly correlated with progression of the disease, overall survival and disease free survival of the patient, hence can be used as predictive markers. Molecular profiles have also been used to provide targeted treatment to the patients. Ertotinib is the FDA approved drug for pancreatic adenocarcinoma patients with upregulated EGFR expression. Combined therapy of dabrafenib and trametinib is preferred treatment for pancreatic cancer patients with BRAF V600E mutation $(46,52)$. Various studies investigating hereditary pancreatic cancers have shown the involvement of BRCA1, BRCA2, CDKN2A, MSH2, MSH6, STK11, PALB2, MLH1, PRSS1, ATM, $A P C$ and PMS2. Attempts have been made for screening families of patients found with alterations in these genes (4). Also, FDA approved drug olaparib is available for use in pancreatic cancer patients with germline BRCA1 mutations (47).

Above investigations clearly show that molecular profiling of cancers play an important role in early diagnosis, prognosis prediction and appropriate treatment selection for pancreatic cancer patients. These factors are significant in improving the survival of these patients. Unfortunately, such researches have not been initiated in Pakistan. Among the 23 pancreatic cancers studies published from Pakistan, none of the study is based on molecular profiling of tumors.

\section{Conclusion}

As discussed above, pancreatic cancer research in Pakistan is not comparable with other parts of the world. Although studies from Pakistan have attempted to present clinical features of pancreatic tumors, data is scattered and inconclusive. The current position of Pakistan in pancreatic cancer research has generated immense need for studies that are based on molecular aspects of the pancreatic tumors with sufficient sample size. Association of clinical characteristics of tumors and patients' survival with genetic, transcriptomic and/or proteomic level alterations will also be important. This will assist in using the appropriate clinical features for prediction of survival and prognosis as well as for providing useful treatment to patients. In future, this data may also lead 


\section{Pancreatic Cancers: A Review of Studies from Pakistan and Comparison with Global Trends}

to the development of sensitive diagnostic tools and targeted drugs for pancreatic cancers.

\section{References}

1. Globocan 2020 [cited 2021 April 30] Available from: https://gco.iarc.fr/today/

2. Sarwar MR, Saqib A. Cancer prevalence, incidence and mortality rates in Pakistan in 2012. Cogent Medicine. 2017;4(1):1288773.

3. Pancreatic Cancer Action Network 2019 [cited 2021 Feb 15] Available from: https://www.pancan.org/facingpancreatic-cancer/about-pancreatic-cancer/types-ofpancreatic-cancer/.

4. Rawla P, Sunkara T, Gaduputi V. Epidemiology of Pancreatic Cancer: Global Trends, Etiology and Risk Factors. World Journal of Oncology. 2019;10(1):10.

5. Din NU, Arshad H, Ahmad Z. Solid pseudopapillary neoplasm of the pancreas: a clinicopathologic study of 25 cases from Pakistan and review of Literature. Annals of Diagnostic Pathology. 2014;18(6):358-62.

5. Fakhar M, Gul M, Rashid S. Antagonistic role of Klothoderived peptides dynamics in the pancreatic cancer treatment through obstructing WNT-1 and Frizzled binding. Biophysical Chemistry. 2018;240:107-17.

6. Burney S, Irfan K, Saif MW, Masud F. Diabetes and pancreatic cancer. Journal of the Pancreas. 2014;15(4):319-21.

7. Burney S, Khawaja KI, Saif MW, Masud F. Chemotherapy and metformin in pancreatic adenocarcinoma and neuroendocrine tumors. Journal of the Pancreas. 2014;15(4):313-6.

8. Parvez T, Parvez B. Screening in genetically induced pancreatic cancer: an update. Pakistan Armed Forces Medical Journal. 2005;55(3):241-6.

9. Parvez T, Parvez B, Abdullah BN, Alharabi TM, Ibrahim MI. Advanced pancreatic cancer: past, present and future of treatment. Pakistan Armed Forces Medical Journal. 2005;55(2):146-55.

10. Qadir MI, Faheem A. miRNA: a diagnostic and therapeutic tool for pancreatic cancer. Critical Reviews in Eukaryotic Gene Expression. 2017;27(3).

11. Wu S, Powers S, Zhu W, Hannun YA. Substantial contribution of extrinsic risk factors to cancer development. Nature. 2016;529(7584):43.

12. Cancer Research UK 2019 [cited 2021 Feb 17] Available from: https://www.cancerresearchuk.org/aboutcancer/causes-of-cancer/inherited-cancer-genes-andincreased-cancer-risk/family-history-and-inheritedcancer-genes.

13. Sarfati D, Koczwara B, Jackson C. The impact of comorbidity on cancer and its treatment. CA: A Cancer Journal for Clinicians. 2016;66(4):337-50.

15. Hussain D, Khan MR, Azami R. Surgical palliation for unresectable pancreatic carcinoma. Journal of Pakistan Medical Association. 2004;54(12):601-4.
16. Tomasello G, Ghidini M, Costanzo A, Ghidini A, Russo A, Barni S, et al. Outcome of head compared to body and tail pancreatic cancer: a systematic review and meta-analysis of 93 studies. Journal of Gastrointestinal Oncology. 2019;10(2):259.

17. Birnbaum DJ, Bertucci F, Finetti P, Birnbaum D, Mamessier E. Head and Body/Tail Pancreatic Carcinomas Are Not the Same Tumors. Cancers. 2019;11(4):497.

18. Yu J, Blackford AL, Dal Molin M, Wolfgang CL, Goggins M. Time to progression of pancreatic ductal adenocarcinoma from low-to-high tumour stages. Gut. 2015;64(11):1783-9.

19. Ahmad Z, Din NU, Minhas K, Moeen S, Ahmed A. Epidemiologic data, tumor size, histologic tumor type and grade, pathologic staging and follow up in cancers of the ampullary region and head of pancreas in 311 Whipple resection specimens of Pakistani patients. Asian Pacific Journal of Cancer Prevention. 2015;16:7541-6.

20. Bilal M, Tariq H, Mamoon N. Whipple resection: concordance between frozen section and permanent section diagnosis of surgical margins. Journal of Ayub Medical College Abbottabad. 2018;30(1):26-9.

21. Haq A. Outcome of surgical management of carcinoma of the pancreas. Annals of King Edward Medical University. 2005;11(4).

22. Khan M, Ashraf MI, Syed AA, Hanif F. Impact of different treatment modalities on the outcome of pancreatic cancer. Journal of the College of Physicians and Surgeons. 2017;27(8):470-4.

23. Pal KMI, Bari H, Nasim S. Pancreaticoduodenectomy: a developing country perspective. Journal of the Pakistan Medical Association. 2011;61(3):232.

24. Qureshi A, Hassan U, Azam M. Morphology, TNM staging and survival with pancreatico-duodenectomy specimens received at Shaukat Khanum Memorial Cancer Hospital and Research Centre, Pakistan. Asian Pacific Journal Cancer Prevention. 2011;12:953-6.

25. Shah MF, Pirzada MT, Nasir I, Malik AA, Farooq U, Anwer AW, et al. Surgical outcome of pancreaticoduodenectomy in pancreatic and periampullary neoplasms. Journal of the College of Physicians and Surgeons. 2017;27(9):559-62.

26. Zahir MN, Jabbar AA. Metastatic pancreatic carcinoma and experience with folfirinox-a cross sectional analysis from a developing country. Asian Pacific Journal Cancer Prevention. 2015;16(14):6001-6.

27. Midha S, Chawla S, Garg PK. Modifiable and nonmodifiable risk factors for pancreatic cancer: a review. Cancer Letters. 2016;381(1):269-77.

28. Bhatti ABH, Yusuf MA, Kazmi SAS, Syed AA. Pancreaticoduodenal resection for malignancy in a lowvolume center: long-term outcomes from a developing country. World Journal of Surgery. 2014;38(10):2506-13. 
29. Dar FS, Bhatti ABH, Dogar AW, Zia H, Ulhaq I, Rana A, et al. Is pancreaticodoudenectomy with vascular resection a safe procedure in developing country? Early outcomes and review of national literature. International Journal of Surgery. 2015;21:8-13.

30. Qureshi A, Hassan U, Loya A, Akhter N, Yusuf A. Diagnostic utility of endoscopic ultrasound guided aspiration cytology in evaluation of pancreatic masses. Journal of the College of Physicians and Surgeons. 2013;23(7):484-6.

31. Sato N, Hasegawa Y, Saito A, Motoi F, Ariake K, Katayose $\mathrm{Y}$, et al. Association between chronological depressive changes and physical symptoms in postoperative pancreatic cancer patients. BioPsychoSocial Medicine. 2018;12(1):13.

32. Baekelandt BM, Fagerland MW, Hjermstad MJ, Heiberg T, Labori KJ, Buanes TA. Survival, complications and patient reported outcomes after pancreatic surgery. Journal of Hepato-biliary-Pancreatic Sciences. 2019;21(3):275-82.

33. Batool S, Malik AA, Bari H, Islam I, Hanif F. Vascular resection and reconstruction in pancreatic tumours. Journal of the College of Physicians and Surgeons. 2018;28(6):485-7.

34. Malik A, Shaikh AR, Shaikh GA. Validity of surgical bypass in unresectable pancreatic cancer. Rawal Medical Journal. 2013;38(2):152-5.

35. Rehman MHU, Khan AA. The ct criteria of unresectability for pancreatic carcinoma. Pakistan Journal of Radiology. 2017;27(2).

36. Fahrmann JF, Bantis LE, Capello M, Scelo G, Dennison JB, Patel N, et al. A plasma-derived protein-metabolite multiplexed panel for early-stage pancreatic cancer. Journal of the National Cancer Institute. 2018;111(4):3729.

37. Malik SS, Malik SA, Zulfiqar MB, Iqbal A. Moderately differentiated ductal Carcinoma pancreas involving periampullary region. Annals of King Edward Medical University. 2015;21(2):119-.

38. Cancer Society of Finland 2019 [cited 2021 Feb 20] Available from: https://www.allaboutcancer.fi/factsabout-cancer/stages/

39. Matsuo K, Mandelbaum RS, Machida H, Purushotham S, Grubbs BH, Roman LD, et al. Association of tumor differentiation grade and survival of women with squamous cell carcinoma of the uterine cervix. Journal of Gynecologic Oncology. 2018;29(6).

40. Carstens JL, De Sampaio PC, Yang D, Barua S, Wang H, Rao A, et al. Spatial computation of intratumoral $\mathrm{T}$ cells correlates with survival of patients with pancreatic cancer. Nature Communications. 2017;8:15095.

41. Canadian Cancer Society 2019 [cited 2021 Feb 20] Available from: https://www.cancer.ca/en/cancerinformation/cancer-101/what-is-cancer/stage-andgrade/?region $=$ on

42. Dhani NC. 2017. Hypoxia, invasion and metastasis in pancreatic cancer (Doctoral dissertation).

43. Yousaf A, Ahmad M, Waheed R, Muslim M, Aurangzeb $\mathrm{M}$, Ahmed $\mathrm{N}$, et al. Surgical outcome of upper gastrointestinal malignancies. Journal of Medical Sciences. 2016;24(1):32-5.

44. McGuigan A, Kelly P, Turkington RC, Jones C, Coleman HG, McCain RS. Pancreatic cancer: a review of clinical diagnosis, epidemiology, treatment and outcomes. World Journal of Gastroenterology. 2018;24(43):4846.

45. Neoptolemos JP, Kleeff J, Michl P, Costello E, Greenhalf $\mathrm{W}$, Palmer DH. Therapeutic developments in pancreatic cancer: current and future perspectives. Nature Reviews Gastroenterology \& Hepatology. 2018;15(6):333.

46. Clinical trial data by National Institute of Health 2021 [cited 2021 Feb 25] Available from: https://clinicaltrials.gov/.

47. National Cancer Institute: National Institute of Health, USA; [cited 2021 April 30] Available from: https://www.cancer.gov/aboutcancer/treatment/drugs/pancreatic.

48. Boyiadzis MM, Kirkwood JM, Marshall JL, Pritchard CC, Azad NS, Gulley JL. Significance and implications of FDA approval of pembrolizumab for biomarkerdefined disease. Journal for Immunotherapy of Cancer. 2018;6(1):1-7.

49. Zhao L, Lee VH, Ng MK, Yan H, Bijlsma MF. Molecular subtyping of cancer: current status and moving toward clinical applications. Briefings in Bioinformatics. 2018;20(2):572-84.

48. Aier I, Semwal R, Sharma A, Varadwaj PK. A systematic assessment of statistics, risk factors, and underlying features involved in pancreatic cancer. Cancer Epidemiology. 2019;58:104-10.

49. Waters AM, Der CJ. KRAS: the critical driver and therapeutic target for pancreatic cancer. Cold Spring Harbor Perspectives in Medicine. 2018;8(9):a031435.

50. Guan M, Bender RJ, Pishvaian MJ, Halverson DC, Tuli $\mathrm{R}$, Klempner SJ, et al. Molecular and clinical characterization of BRAF mutations in pancreatic ductal adenocarcinomas (PDACs). American Society of Clinical Oncology; 2018;36(4):214. 